Brit. F. vener. Dis. (1973) 49, 148

\title{
Changing patterns in the organization of the venereal diseases service in Portugal
}

\author{
F. NORTON BRANDÃO \\ Central Dispensary of Social Hygiene, Lisbon, Portugal
}

The Ministry of Health and Social Security in Portugal was reorganized in 1971. Before this the Board of General Health Direction carried out the task of fighting venereal diseases through Central Dispensaries of Social Health in the three most important towns: Lisbon, Oporto, and Coimbra, as well as through smaller health centres all over the country.

The Central Dispensaries of Social Health had administrative autonomy and undertook antivenereal disease campaigns through units located for the convenience of the general public, and were responsible for patient care, laboratory diagnosis, and treatment, all of which were free.

Cultures for Neisseria gonorrhoeae, Trichomonas vaginalis, and Candida albicans as well as serological tests for syphilis were undertaken at the Central Laboratories. Cooperating with these medical services there was a Board of Social Assistance and Epidemiology. The Central Dispensaries of Social Health had special independent units for the control of clandestine prostitutes and arranged their admission to hospital when necessary. These units included social rehabilitation services which were run by the French organization Nid. These Central Dispensaries also carried out prophylaxis programmes against blindness, cancer, ringworm, and dental caries.

In the smaller towns, where venereal diseases are less common, antivenereal measures were in the hands of local health officers.

When the Ministry of Health and Social Security was reorganized in 1971 to promote comprehensive medical care for the general public, it was split up into two departments for teaching and research and three Boards of General Direction: Health, Hospitals, and Social Assistance.

The Central Dispensaries and Local Health Centres were integrated respectively with District and Municipal Health Centres, which are supervised by the General Health Board.

Besides their work in controlling venereal disease, the District and Municipal Centres are also responsible for the following:

-Occupational and community health

- Maternal and child care and welfare

- Prophylaxis of certain preventable diseases and vaccination centres

- Mental health

-Public health nurses

- Public health education

- Social services

- Statistics

In addition, the District Centres are also responsible for the following:

-Dental care

-Care of the blind

- Care of the deaf

- Cancer prophylaxis

-Public health laboratory services

Working in close collaboration with the Centres are committees which coordinate the health services and social welfare. 